\title{
Correction to: Detection of hysteroscopic fluid in the pouch of Douglas: a prospective cohort study about the predictability of bilateral tubal occlusion
}

\author{
Marlene Hager ${ }^{1}$. Johannes Ott ${ }^{1}$ (1) $\cdot$ Christian Göbl $^{2} \cdot$ Iris Holzer $^{1} \cdot$ Rudolf Seemann $^{3}$. Christine Kurz ${ }^{1}$. \\ John Preston Parry ${ }^{4,5}$
}

Published online: 19 May 2021

(c) Springer-Verlag GmbH Germany, part of Springer Nature 2021

\section{Correction to: Archives of Gynecology and Obstetrics https://doi.org/10.1007/s00404-021-05993-0}

In the Methods section, the following sentence is incorrect: "This analysis used odds ratios with $95 \%$ confidence intervals (95\% CI), the Wald-test, and the likelihood ratio (LR) test." The correct sentence is "This analysis used odds ratios with $95 \%$ confidence intervals (95\% CI) and the Wald-test.".

Accordingly, in Table 4, the headlines of two rows must be re-named: "p (Wald's test)" should be "test statistic" and the "p (LR test)". Moreover, in Table 2, the correct median fluid shift for "Unilateral tubal occlusion $(\mathrm{n}=22)$ " is 2.7 $(0.0 ; 5.3)$.

Publisher's Note Springer Nature remains neutral with regard to jurisdictional claims in published maps and institutional affiliations.

The original article can be found online at https://doi.org/10.1007/ s00404-021-05993-0.

Johannes Ott

johannes.ott@meduniwien.ac.at

1 Clinical Division of Gynecological Endocrinology and Reproductive Medicine, Medical University of Vienna, Spitalgasse 23, 1090 Vienna, Austria

2 Clinical Division of Obstetrics and Feto-Maternal Medicine, Department of Obstetrics and Gynecology, Medical University of Vienna, Vienna, Austria

3 Department of Oral and Maxillofacial Surgery, Medical University of Vienna, Vienna, Austria

4 Parryscope ${ }^{\circledR}$ and Positive Steps Fertility, Madison, MS, USA

5 Department of Obstetrics and Gynecology, University of Mississippi Medical Center, Jackson, MS, USA 